\title{
Integration of the trans-operative information with the patient's electronic record
}

\author{
By E. K. Souza', A. M. Marciano² \\ ${ }^{1}$ Moinhos de Vento Hospital/ Clinical Engineering, Porto Alegre, Brazil \\ ${ }^{2}$ Moinhos de Vento Hospital/ Clinical and Hospital Engineering, Porto Alegre, Brazil
}

\begin{abstract}
This article presents an integration project between the anesthetic station used in the step of trans-operative (life signals multiparameter monitor, anesthesia device and controlled-target infusion pump) and the system of hospital information. The main goal of this project is to automatically capture the vital signals from the medical equipment and the records trans-operatives and provide an anesthesia record to be stored in the patient's electronic medical record. The integration mode is through a gateway that executes the conversion of the machine- specific language into data/information of the HL7 standard. This interaction will allow integrating data and information from multiparametric monitors, anesthesia devices, Controlled-target infusion pumps, and the intra-operative anesthesiologist inputs.

Keywords - Medical Equipment, Anesthesia Station, Step of the trans-operative, Patient Data Integration, Electronic Records.

Copyright (C) 2021. This is an open-access article distributed under the terms of the Creative Commons Attribution License (CC BY): Creative Commons - Attribution 4.0 International - CC BY 4.0. The use, distribution or reproduction in other forums is permitted, provided the original author(s) and the copyright owner(s) are credited and that the original publication in this journal is cited, in accordance with accepted academic practice. No use, distribution or reproduction is permitted which does not comply with these terms.
\end{abstract}

\section{INTRODUCTION}

The patient's record is a mandatory document in Health Assistance Establishment and is considered an extremely important tool that requires a mechanism of follow up of the data inserted in it. The electronic medical record can be quoted as one of the mechanisms that present many advantages to the institution such as paper use reduction, digital certification, more reliability and safety for the data inserted in the records and, to the information derived from it. ${ }^{1}$

It has been claimed that a combination of Information Technology (IT) devices such as computers, communication networks, medical information, and online electronic data can improve the quality and decisions concerning health care. Therefore, the automation of the patient's records (even incomplete) contributes significantly to the quality improvement of the treatment beyond the effective control of costs involved. ${ }^{2}$ A medical record system is represented by a series of components that form mechanisms so the records can be created, used, stored, and accessed as part of a hospital information system (HIS). ${ }^{3}$

In the surgical environment, there are examples of software development that consists of the data integration of monitoring parameters during anesthesia and a group of rules configured by the anesthesiologist. Alerts generated by the software provide important information about the patient's condition and eventual risk situations which wouldn't be highlighted if only the individual alarms of the parameters coming from the monitors were considered. ${ }^{4}$ 
This project presents an integration proposition between the anesthesia station equipment used in the trans-operative stage (multiparametric monitors, anesthesia devices, controlled-target infusion pumps) and the patient's medical electronic records (PEMR). The goal is to automatically capture the vital signals from the medical equipment and the records of the trans-operative data to create anesthesia file that integrate the PEMR with the HIS. The specific goals of this project are: (a) increase the safety and reliability of data and information from the trans-operative stage; (b) qualify this data and information via automatic inputs with no further manipulation; (c) improve the medical document and protocol pattern; (d) diminish the probability of failures and increase clarity; (e) facilitate the data research and information for studies, benefiting doctors, hospitals, and patients.

\section{METHODS}

Described below are the development phases of the project, the integration alternatives, the integration method option used, and the information that will be integrated into the implementation stage.

\section{A. Project Development}

The stages of development flow-gram of the project are described below in Figure 1.

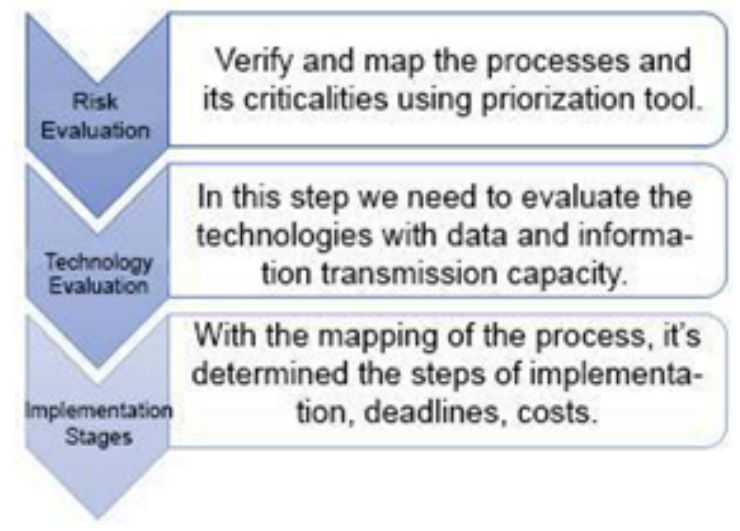

FIGURE 1. Project development flow-gram.

As demonstrated in Figure 1, risk evaluations were executed as well as the vulnerability/fragility of the anesthetic procedure related to the absence of automated records and data integration and information from the medical technology used in this stage of the surgical process. Next, we surveyed the hospital's equipment that can already transmit data for then plan and the sub-stages of the implementation. The next stage consisted of the processes/operations/activities detailing the peculiarities that determine which data and information would be registered and integrated, the technologies available in the market for this type of record integration as well as the costs.

\section{B. Architecture/Topology of the Integration System}

The definition of interoperability, according to the Institute of Electric and Electronic Engineers (IEEE) deals with the capacity of two or more systems to exchange information and being able to use the information exchanged. ${ }^{5}$ The communication Health Level 7 (HL7) is a communication structure with determined standards to the exchange execution, integration and sharing of communication information between devices or clinical data system devices. ${ }^{6}$

The architectures or topologies of communication can be divided into two suggested formats, "without Gateway" and "with Gateway." In the topology format without gateway, the data are transmitted from equipment in HL7 and are processed before storage in HIS. The format with Gateway requires an intermediary device that physically separates the HIS and the biomedical equipment. This device performs the machine-specific language conversion of HL7 standard to be consequently processed and stored. Concerning the integration method of monitoring data and trans-operative stage information, the topologies and/or architectures are demonstrated in Figures 2 and 3.

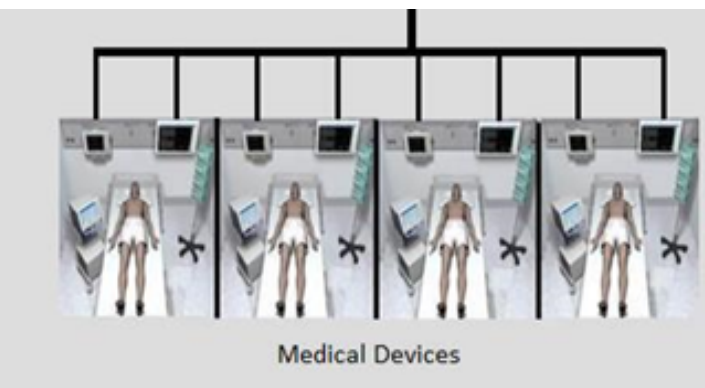

FIGURE 2. "Direct" topology without Gateway. 
In the architecture model of Figure 2, the equipment provides the information directly in the HL7 language allowing the storage of clinical data in the HIS database. In Figure 3 below, it is demonstrated the topology using the intermediary device, named Gateway.

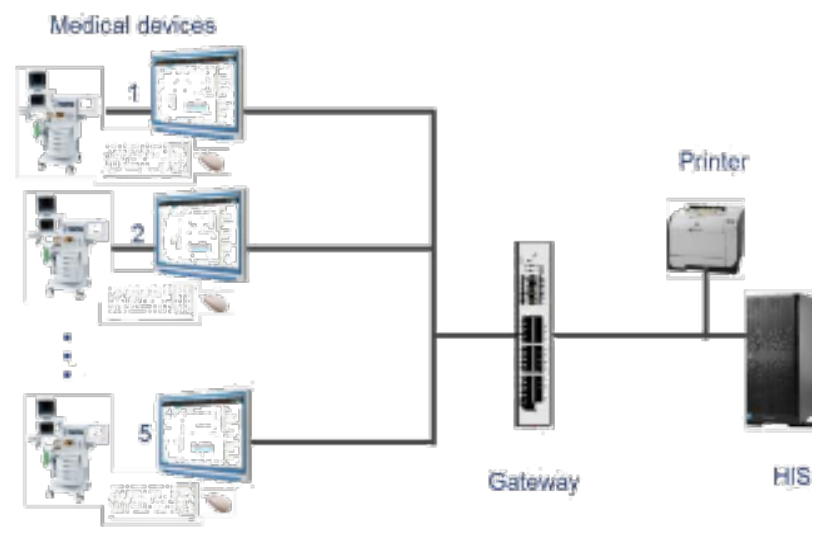

FIGURE 3. "Indirect" topology with Gateway.

In this type of topology, the information from the equipment needs a data converter named Gateway responsible for trans-coding the information from the equipment to clinical storage data in PEMR.

In this last model evaluated it is observed the need of more devices to integrate and intercommunicate data treatment and information storage appliance so the information generates an electronic anesthesia file with further storage in PEMR.

Considering the evaluated options of integration methods and equipment profiles installed in hospitals it was necessary to use the topology demonstrated in Figure 4.

\section{RESULTS}

The suggested method was applied in a private, nonprofit hospital that has around 400 hospital beds and is located in the capital of Rio Grande do Sul (a Brazilian State). The hospital has around 4500 pieces of biomedical equipment.

Among the various areas that execute assistance health care treatment, using technologies, was defined the automatic capture of information from the trans-operative process from the equipment that was able to transmit data referring to the patients' monitoring and therapy, in
5 specific surgical rooms to store in PEMR and HIS. The topology to be used in this project is illustrated in Figure 4.

The data to be captured and information inserted in this integration are related in Table 1.

TABLE 1. Integration Parameters

\begin{tabular}{|c|c|}
\hline Origin & Information \\
\hline $\begin{array}{c}\text { Multi - parameter } \\
\text { Monitor }\end{array}$ & Vital Signals (ECG, SpO2, PNI, PI, CO2) \\
\hline Anesthesia Equipment & $\begin{array}{c}\text { Ventilator Data and Anaesthetic Gases } \\
\text { (Vmin, Aereal vias pressure, frequency, } \\
\text { PEEP, ventilator mode) }\end{array}$ \\
\hline $\begin{array}{c}\text { Controlled Target } \\
\text { Infusion Pumps Drugs }\end{array}$ & $\begin{array}{c}\text { Volume and administered concentrations } \\
\text { Anaesthesiologist Input }\end{array}$ \\
\hline
\end{tabular}

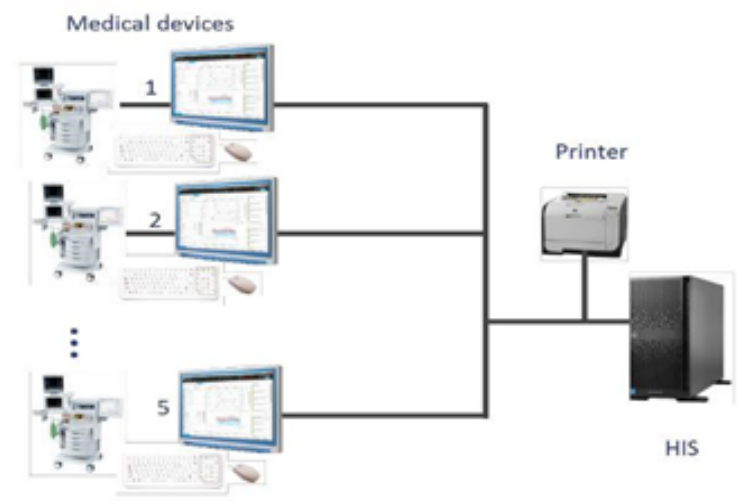

FIGURE 4. Topology used in the project (without Gateway).

In the architecture of Figure 4, the data of the anesthesia station provided by the equipment (multiparametric monitors, anesthesia devices, Controlled target Infusion pumps) and the information and events manual input in the trans-operative (executed by the anesthesiologist) are integrated and converted in HL7 by an All in One Computer (AOC) attached to the anesthesia activities medical-assistance device, providing the institution the information to improve its procedures.

As described in Table 1, this integration allows to automatically register data from the patient's vital signals in surgery, ventilator mechanics, anesthetic gases, drugs 
infused, as well as the events and information trans-operatively executed and informed by the anesthesiologist.

It was also possible to identify which types and quantities of equipment are integrated with further stages so the solution can be implemented in all surgical rooms in the hospital, and thus allow the estimation of the financial resources needed to invest in future implementation to include budget planning in the institution's posterior year.

\section{DISCUSSION}

This project is a consequence of the maturing associated with the continuous improvement of the storage processes of the patient information from the service promoted by a large hospital in the south region of Brazil.

It's worth pointing out that the success of this project is directly tied to the active participation of the Medical, Technology Information, Clinical Engineering teams beyond investing in needed hospital equipment that can export data, besides in technology information infrastructure including processing and storage servers, cabling, enabled network points, etc.

Attention is needed concerning issues including concept alignment with the board of directors and scenario evaluation referring to opening the service suggested (because this project will change the modus operandi). Another important factor is in the previous capacity of all the teams involved.

The data safety has worried various sectors in many areas in the world, therefore, it is recommended that the product selection and technologies have the recognition of the National Health Surveillance Agency (ANVISA) or similar group in the application country and are in compliance with the Information Safety Rules.

The continuity of application of this integration, the way the data and information are transferred, stored and further accessed, and the safety protocols, are susceptible to further analysis, developments, adjustments, and validations.

\section{CONCLUSION}

The health organizations using electronic systems tend to have more effective control over data and patients' clinical information. This more efficient way of information collection can provide safety, transparency, and better service to the patient, allowing the audit of activities such as medical-assistance, providing the institution data to improve its procedures.

In more advanced centers and some Brazilian hospitals this form of more efficient collection, storage, and information integration begins to develop, mainly in the application of intense therapy unities. ${ }^{7}$

The clinical engineering teams with their multidisciplinary knowledge can contribute to the medical teams, assistance, and information technology and become increasingly applied to the integrated possible technologies.

The expectation is that the project will automatically capture the vital signals from the medical equipment and the records from the trans-operative and to provide an anesthesia file to be stored in the PEMR and in HIS, which can effectively contribute to the safety and reliability of data and information from the trans-operative stage. The project will also qualify the data and information via the automatic inputs and with no further manipulation.

This will contribute to improving the standardization of documents and medical protocols, decrease failures, and provide more clarity in adverse events via the ease of data search and information for studies which benefits doctors, assistants, hospitals, and patients.

\section{CONFLICT OF INTEREST}

The authors declare that they have no conflict of interest.

\section{REFERENCES}

1. ENADE, Tecnologia em Gestão Hospitalar, 2016.

2. Lindberg DAB and Humphreys BL. Computers in medicine. J Am Med Assoc 1995;272(231)1667-68.

3. Rodrigues F, Xavier J, Jefferson Colombo B, and Adriano AL. A tecnologia da informação na área hospitalar: um caso de implementação de um sistema de registro de pacientes.Rev. adm. contemp.[online]. 2001;5(1)10520. ISSN 1982-7849. http://dx.doi.org/10.1590/ S1415-65552001000100007. 
4. Dherte PM et al. Alertas Inteligentes: desenvolvimento de software para otimização dos dados de monitorização. Revista Brasileira de Anestesiologia 2011;61(1).

5. Moreno RA. Interoperabilidade de Sistemas de Informação em Saúde. J Health Informati 2016;8(3).
6. Stiefel $\mathrm{R}$ and Riskalla E. The elements of a complete product evaluation. 1995. Available at http://www. hl7.org/about/index.cfm?ref=common.

7. Rebelo M, et al. vMon-mobile: experiência na integração de monitores de sinais vitais ao PEP utilizando tecnologia de redes sem fio. Serviço de Informática, Instituto do Coração (Incor) HCFMUSP, Brasil. 